\title{
夏玉米光合特性及光响应曲线拟合
}

\author{
李 力 $^{1}$ 张祥星 $^{1}$ 郑 ${\text { 睿 } 1,2^{*}}^{\text {郭建青1,2 }}$ \\ ${ }^{1}$ 长安大学环境科学与工程学院, 西安 $710054 ;{ }^{2}$ 早区地下水文与生态效应教育部重点实验室, 西安 710054
}

\begin{abstract}
摘 要 通过对光合-光响应曲线的研究来探索光合有效辐射与净光合速率的关系是一种非常重要的手段。合适的模型才能 较好地反映植株的光合特性。分析由于生育期与叶位的不同而导致各模型拟合值与实测值差异的变化性以及不同光合有效辐 射强度下各模型的适用性, 可为夏玉米(Zea mays)光合模型的选择和光合-光响应曲线模型的进一步优化提供参考。该研究运 用3种典型的光合模型对夏玉米大喇叭口期与乳熟期上部叶、穗位叶与下部叶做光合-光响应曲线拟合, 对比各模型的拟合度

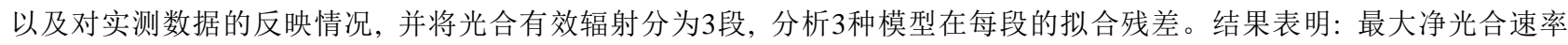
$\left(P_{\mathrm{nmax}}\right) 、$ 饱和光强 $\left(I_{\mathrm{sat}}\right) 、$ 暗呼吸速率 $\left(R_{\mathrm{d}}\right)$ 等光合特征参数值随叶位由上而下呈降低趋势, 乳熟期普遍小于大喇叭口期; 不同生 育期和叶位 3 种模型均可以拟合, 直角双曲线模型、非直角双曲线模型对 $P_{\mathrm{nmax}} 、 I_{\mathrm{sat}}$ 的拟合值与实测值有一定的偏差; 通过残 差分析表明叶子飘模型的拟合值与实测值最为相符, 尤其对高光部分的拟合表现出独有的优越性。
\end{abstract}

关键词 光合特征参数; 叶子飘模型; 饱和光强; 残差

引用格式: 李力, 张祥星, 郑睿, 郭建青 (2016). 夏玉米光合特性及光响应曲线拟合. 植物生态学报, 40, 1310-1318. doi: 10.17521/cjpe.2016.0082

\section{Photosynthetic characteristics and photosynthesis-light response curve models of summer maize}

LI Li ${ }^{1}$, ZHANG Xiang-Xing ${ }^{1}$, ZHENG Rui ${ }^{1,2 *}$, and GUO Jian-Qing ${ }^{1,2}$

${ }^{1}$ School of Environmental Science \& Engineering, Chang'an University, Xi'an 710054, China; and ${ }^{2}$ Key Laboratory of Subsurface Hydrology and Ecological Effect in Arid Region of Ministry of Education, Xi'an 710054, China

\section{Abstract}

Aim The photosynthesis-light response curve is the most commonly used method to explore the relationship between photosynthetically active radiation and the net photosynthetic rate, because it is more effective to reflect the plant photosynthetic characteristics. And it is very meaningful for researchers to choose a suitable summer corn (Zea mays) photosynthetic model and optimal the models of photosynthesis-light response curve by analyzing the differences between simulation and observation in each growth period of some plant. So the object of this paper was to propose some useful suggestions for the choice of summer corn photosynthetic modes and the optimization of the photosynthetic light response curves model in further.

Methods In this paper, three typical photosynthetic models were used to fitting the photosynthetic light response curve for upper leaf, leaf at ear of grain and lower leaf of summer corn during bell and milk period. And then the fitting degree of each model was compared to the measured data. Photosynthetic active radiation was divided into three parts, and the fitting residual errors of these three models were analyzed individually.

Important findings The photosynthetic characteristic parameters such as maximum net photosynthetic rate $\left(P_{\mathrm{nmax}}\right)$, saturated light intensity $\left(I_{\mathrm{sat}}\right)$ and dark respiration rate $\left(R_{\mathrm{d}}\right)$ decreased constantly with a top-down leaf position and the parameters at milk stage were generally lower than the bell stage. Each growth period and leaf position could fit the curve, but some deviation exists for the $P_{\text {nmax }}$ and $I_{\text {sat }}$ in the rectangular hyperbolic model and the non-rectangular hyperbolic model. The results of curve fitting residual showed that the simulation values from Ye Zi-Piao model were closest to the actual values, and especially for the high photosynthetically active radiation section.

Key words photosynthetic characteristic parameters; Ye Zi-Piao model; saturated irradiance; residue

Citation: Li L, Zhang XX, Zheng R, Guo JQ (2016). Photosynthetic characteristics and photosynthesis-light response curve models of summer maize. Chinese Journal of Plant Ecology, 40, 1310-1318. doi: 10.17521/cjpe.2016.0082

收稿日期Received: 2016-03-04 接受日期Accepted: 2016-09-21

* 通信作者Author for correspondence (E-mail: rayzheng1986@gmail.com) 
光合作用是生物界赖以生存的基础, 光是光合 作用的主导因子, 对每种绿色植物均可做出光合作 用对光的响应曲线(刘宇锋等, 2005)。通过对光合光响应曲线的研究来探索光合有效辐射与净光合速 率的关系是一种非常重要的手段。Baly (1935)提出 了用直角双曲线模型拟合光合光响应曲线, 随后出 现非直角双曲线模型(Thornley, 1976)、指数函数模 型(Bassman \& Zwier, 1991)以及由我国学者叶子飘 提出的模型(Ye, 2007, 以下称叶子飘模型)等。广大 学者应用这些模型对各类植物的光合-光响应曲线 进行拟合从而研究植物的光合特性。如用指数函数 模型对扁宿豆(Melissitus ruthenica)光合特性的研究 (王照兰等, 2009)、用叶子飘模型研究温度变化对烟 草(Nicotiana tabacum)光合作用光响应特征的影响 (钟楚等, 2012)等等。在拟合过程中不同模型具有其 各自的优缺点, 合适的模型才能更好地拟合光合特 征参数, 在以往对某种植物做不同的水分、施肥量 或品种下各模型的光合-光响应曲线拟合时, 忽略 了由于生育期与叶位的不同而导致各模型拟合值与 实测值差异的变化性以及不同光合有效辐射下各模 型的适用性; 残差作为实测数据与回归估计值的差 值, 通过拟合残差所提供的信息, 分析拟合数据的 可靠性、周期性或其他干扰已被广泛应用于天文、 气象和光学等各个领域(周朴等, 2010; 陈华华等, 2013; 孙乐强等, 2014)。为了探讨光合-光响应模型 对不同生育期不同叶位拟合情况的差异性, 采用3 种模型对夏玉米(Zea mays)做光合-光响应曲线拟 合, 对比分析不同生育期与叶位的拟合结果, 并结 合残差分析不同光合有效辐射下各模型的优缺点与 适应性, 为夏玉米拟合模型的选择和光合-光响应 曲线模型的进一步优化提供参考。

\section{1 材料和方法}

\section{1 实验材料}

实验场地位于西北农林科技大学旱区农业水土 工程教育部重点实验室塑胶大棚内, 选择抗虫性高 的夏玉米品种 ‘农大375’, 于2015年6月18日在塑料 膜透光大棚内种植20盆, 塑料盆上部内口直径31.8 $\mathrm{cm}$, 高34 $\mathrm{cm}$ 。全生育期123天, 幼苗期与抽雄期进 行两次施肥, 采用传统的称质量法与EM50土壤水 分监测仪(DECAGON, Pullman, USA)对盆内水分监 测。浇水上限为 $90 \%$ 田间持水量, 下限为 $70 \%$ 田间持
水量, 为充分供水环境。分别在2015年7月31日至8 月 2 日和 9 月 13 日至 9 月 15 日进行光合-光响应曲线的 测定, 随机选择3株长势均匀一致的健壮植株进行 测定。根据拔节期测量的叶倾角度数自上往下分别 选取叶倾角小于 $10^{\circ}$ 、小于 $20^{\circ}$ 、小于 $30^{\circ}$ 的第一个叶 片, 依次标记为上部叶、穗位叶、下部叶。测量仪 器采用LI-6400便携式光合测定仪(LI-COR, Lincoln, USA), $\mathrm{CO}_{2}$ 浓度设置为 $380 \mu \mathrm{mol} \cdot \mathrm{mol}^{-1}$, 温度设置为 $33{ }^{\circ} \mathrm{C}$, 光合有效辐射梯度设置为 $2000 、 1800$ 、 $1600 、 1400 、 1200 、 1000 、 800 、 600 、 400 、 200 、$ 100、 $50 、 0 \mu \mathrm{mol} \cdot \mathrm{m}^{-2} \cdot \mathrm{s}^{-1}$ 。 测量前让待测定叶片在 光合光量子通量密度为 $1200 \mu \mathrm{mol} \cdot \mathrm{m}^{-2} \cdot \mathrm{s}^{-1}$ 的光下、 空气中诱导约 $60 \mathrm{~min}$, 待叶片光合诱导期结束后, 开始测量。

\section{2 光合光响应曲线模型回顾}

不同模型的数学方程以及计算光合特征参数的 方法是不同的, 在运用这些模型拟合时, 需要先对 数学模型以及计算参数时所做出的假设做简单介 绍。

直角双曲线模型(Baly, 1935):

$$
P_{\mathrm{n}}=\frac{\alpha I P_{\mathrm{nmax}}}{\alpha I+P_{\mathrm{nmax}}}-R_{\mathrm{d}}
$$

非直角双曲线模型(Thornley, 1976):

$$
P_{\mathrm{n}}=\frac{\alpha I+P_{\mathrm{nmax}}-\sqrt{\left(\alpha I+P_{\mathrm{nmax}}\right)^{2}-4 \theta \alpha I P_{\max }}}{2 \theta}-R_{\mathrm{d}}
$$

叶子飘模型(Ye, 2007)

$$
P_{\mathrm{n}}=\alpha \frac{1-\beta I}{1+\gamma I}-R_{\mathrm{d}}
$$

式中 $P_{\mathrm{n}}$ 为净光合速率, $I$ 为光强, $\alpha$ 为植物光合作用对 光响应曲线在 $I=0$ 时的斜率, 即初始量子效率, $P_{\mathrm{nmax}}$ 为最大净光合速率, $R_{\mathrm{d}}$ 为暗呼吸速率, $\theta$ 为反映 光响应曲线弯曲程度的曲角参数, 取值 $0<\theta \leqslant 1$, $\beta$ 为光抑制项, $\gamma$ 为光饱和项。用公式(1)、(2)计算时 需要估算植物的饱和光强, 做法为: 用直线方程拟 合弱光强条件下 $\left(\leqslant 200 \mu \mathrm{mol} \cdot \mathrm{m}^{-2} \cdot \mathrm{s}^{-1}\right)$ 的光响应数 据得到表观量子效率 $(A Q E)$, 利用非线性最小二乘 法估算 $P_{\mathrm{nmax}}$, 而对于饱和光强 $\left(I_{\mathrm{sat}}\right)$, 需要由直线方 程 $P_{\mathrm{n} \text { max }}=A Q E \times I_{\mathrm{sat}}-R_{\mathrm{d}}$ (叶子飘, 2010)求解。方程(3) 是有极值的函数, 可以直接求解 $P_{\mathrm{nmax}} 、 I_{\mathrm{sat}}$ 的解析解。

\section{3 数据处理}

实验数据处理由Excel完成, 作图软件为Origin 8.0 , 显著性分析由SPSS 19完成。 


\section{2 结果}

\section{1 光合特征参数实测数据分析}

叶龄是生命活动长短的标尺, 叶龄以及生长状 况不同，会直接影响植株光合作用与光合特征参数 (魏雅芬等, 2011; 陈凯利等, 2013), 取两生育期各 部位叶片多组实测数据的平均值对其光合特征参数 进行分析(表1)。

两生育期不同部位叶片光合特征参数有明显的 差异: 在大喇叭口期, $A Q E$ 表现为上部叶>穗位叶> 下部叶，降幅依次为6.6\%、10.5\%; $P_{\mathrm{n} \max }$ 上部叶与穗 位叶差别不大, 下部叶较穗位叶降低 $34.7 \% ; R_{\mathrm{d}}$ 表现 为上部叶 $>$ 穗位叶 $>$ 下部叶。在乳熟期, $A Q E$ 表现为 上部叶>穗位叶>下部叶，降幅依次为 $20.8 \% 、 4.8 \%$; $P_{\mathrm{nmax}}$ 表现为上部叶 $>$ 穗位叶 $>$ 下部叶, 降幅依次为 $5.3 \% 、 11.9 \% ; R_{\mathrm{d}}$ 表现为上部叶>穗位叶>下部叶，降 幅依次为 $22.3 \% 、 13.8 \%$ 。不同生育期叶片的光合特 征参数也存在差异, 乳熟期较大喇叭口期各部位叶 片的光合特征参数基本均呈现下降趋势, 上部叶 $A Q E$ 下降 $13.1 \%$, 穗位叶 $A Q E$ 下降 $26.3 \%$, 下部叶 $A Q E$ 下降 $21.6 \%$; 上部叶 $P_{\mathrm{n} \text { max }}$ 下降 $46.4 \%$, 穗位叶 $P_{\mathrm{nmax}}$ 下降 $49.6 \%$, 下部叶 $P_{\mathrm{n} \text { max }}$ 下降 $32.5 \%$; 上部叶 $R_{\mathrm{d}}$ 下降 $33.3 \%$ 、穗位叶 $R_{\mathrm{d}}$ 下降 $46.5 \%$, 下部叶 $R_{\mathrm{d}}$ 下降 $43.0 \%$ 。

分析发现：叶片的 $A Q E 、 P_{\mathrm{nmax}}$ 与 $R_{\mathrm{d}}$ 等光合特征 参数均是由叶位自上而下逐渐降低的, 乳熟期到大 喇叭口期叶片的光合特征参数值也是下降的, 后者 降幅更大, $A Q E$ 表示植株对光能尤其是弱光的利用
效率(郎䒯等, 2011)，一定环境条件下，叶片 $P_{\mathrm{n} \max }$ 代 表了其最大光合能力(陆佩玲等, 2000), 说明叶位自 上而下叶片的光能利用率与最大光合能力都在降 低, 大喇叭口期光能的利用率与最大光合能力大于 乳熟期。暗呼吸产生能量(腺苷三磷酸、还原型烟酰 胺腺嘌呤二核苷酸)、碳骨架为植物生理活动提供了 能量和物质基础(蒋高明, 2004), 乳熟期较低的 $R_{\mathrm{d}}$ 可 以降低呼吸作用对光合产物的消耗，有利于干物质 的累积(张其德等, 2001)。

\section{2 光合-光响应曲线模型}

在时间上(同部位叶片不同生育期)与空间上(同 生育期不同部位)叶片的光合特性均有差异，那么 这种差异对光合-光响应曲线拟合模型有何影响? 为了探讨这一问题，运用直角双曲线模型、非直角 双曲线模型及叶子飘模型分别对大喇叭口期与乳熟 期不同部位叶片进行光合-光响应曲线拟合，拟合 结果见表2。

3种模型的决定系数均较高, 最低值 0.996 , 初 始量子效率 $(\alpha)$ 在 $0-0.125$ 理论范围值之内(陆佩玲 等, 2000)。说明直角双曲线模型、非直角双曲线模 型、叶子飘模型都可以用来拟合玉米不同生育期不 同部位叶片的光合-光响应曲线(图1)。但衡量一个模 型的适用性不是仅仅看拟合度的高低，还要结合拟 合参数对实测值的反映情况，对比分析拟合结果， 各模型拟合的参数值有差异, 对实测值的反映情况 也不尽相同，由叶子飘模型得到的 $P_{\mathrm{n} m a x}$ 的拟合值与 实测值最接近，直角双曲线模型与非直角双曲线模

表1 玉米大喇叭口期与乳熟期光合特征参数

Table 1 Photosynthetic characteristic parameters of Zea mays at bell stage and milk stage respectively

\begin{tabular}{|c|c|c|c|c|c|c|}
\hline 时期 Period & 叶位 Leaf position & $\begin{array}{l}\text { 表观量子效率 } A Q E \\
\left(\mu \mathrm{mol} \mathrm{CO}_{2} \cdot \mu \mathrm{mol}^{-1} \text { photons }\right)\end{array}$ & $\begin{array}{l}\text { 最大净光合速率 } P_{\mathrm{nmax}} \\
\left(\mu \mathrm{mol} \cdot \mathrm{m}^{-2} \cdot \mathrm{s}^{-1}\right)\end{array}$ & $\begin{array}{l}\text { 饱和光强 } I_{\mathrm{sat}} \\
\left(\mu \mathrm{mol} \cdot \mathrm{m}^{-2} \cdot \mathrm{s}^{-1}\right)\end{array}$ & $\begin{array}{l}\text { 光补偿点 } I_{\mathrm{c}} \\
\left(\mu \mathrm{mol} \cdot \mathrm{m}^{-2} \cdot \mathrm{s}^{-1}\right)\end{array}$ & $\begin{array}{l}\text { 暗呼吸速率 } R_{\mathrm{d}} \\
\left(\mu \mathrm{mol} \cdot \mathrm{m}^{-2} \cdot \mathrm{s}^{-1}\right)\end{array}$ \\
\hline \multirow{7}{*}{$\begin{array}{l}\text { 大喇叭口期 } \\
\text { Bell stage }\end{array}$} & 上部叶 & $0.061^{\mathrm{a}}$ & $36.78^{\mathrm{a}}$ & $2000^{\mathrm{a}}$ & $48.87^{\mathrm{a}}$ & $3.36^{\mathrm{a}}$ \\
\hline & Upper leaf & & & & & \\
\hline & 穗位叶 & $0.057^{\mathrm{b}}$ & $36.99^{\mathrm{a}}$ & $2000^{\mathrm{a}}$ & $49.14^{\mathrm{a}}$ & $3.25^{\mathrm{a}}$ \\
\hline & Leaf at ear of grain & & & & & \\
\hline & 下部叶 & $0.051^{\mathrm{c}}$ & $24.20^{\mathrm{b}}$ & $1800^{\mathrm{b}}$ & $43.40^{\mathrm{b}}$ & $2.63^{\mathrm{b}}$ \\
\hline & Lower leaf & & & & & \\
\hline & $\begin{array}{l}\text { 上部叶 } \\
\text { Upper leaf }\end{array}$ & $0.053^{\mathrm{a}}$ & $19.70^{\mathrm{a}}$ & $1800^{\mathrm{a}}$ & $32.29^{\mathrm{a}}$ & $2.24^{\mathrm{a}}$ \\
\hline \multirow{3}{*}{$\begin{array}{l}\text { 乳熟期 } \\
\text { Milk stage }\end{array}$} & 穗位叶 & $0.042^{\mathrm{b}}$ & $18.66^{\mathrm{a}}$ & $1800^{\mathrm{a}}$ & $32.32^{\mathrm{a}}$ & $1.74^{\mathrm{b}}$ \\
\hline & Leaf at ear of grain & & & & & \\
\hline & $\begin{array}{l}\text { 下部叶 } \\
\text { Lower leaf }\end{array}$ & $0.040^{\mathrm{c}}$ & $16.44^{\mathrm{b}}$ & $1800^{\mathrm{a}}$ & $31.43^{\mathrm{a}}$ & $1.50^{\mathrm{c}}$ \\
\hline
\end{tabular}

根据最小显著差异法，表中同一列不同字母表示在 0.05 水平上处理间差异显著。

Different letters in same column indicate significant difference at 0.05 level according to least significant difference. AQE, apparent quantum yield of photosynthesis; $P_{\text {nmax }}$, maximum net photosynthetic rate; $I_{\text {sat }}$, satuation light intensity; $I_{\mathrm{c}}$, light compensation point; $R_{\mathrm{d}}$, dark respiration rate. 
表2 3种光响应曲线模型拟合大喇叭口期与乳熟期光响应曲线的实测值与拟合结果

Table 2 Simulation results of light response parameters fitted by three models and the measured data at bell stage and milk stage respectively

\begin{tabular}{|c|c|c|c|c|c|c|c|c|}
\hline $\begin{array}{l}\text { 时期 } \\
\text { Period }\end{array}$ & $\begin{array}{l}\text { 叶位 } \\
\text { Leaf } \\
\text { position }\end{array}$ & $\begin{array}{l}\text { 模型 } \\
\text { Model }\end{array}$ & $\begin{array}{l}\text { 初始量子效率 } \\
\alpha{\text { ( } \mu \mathrm{mol} \mathrm{CO}_{2}}^{-1} \\
\left.\mu \mathrm{mol}^{-1} \text { photons }\right)\end{array}$ & $\begin{array}{l}\text { 最大净光合速率 } \\
P_{\mathrm{nmax}} \\
\left(\mu \mathrm{mol} \cdot \mathrm{m}^{-2} \cdot \mathrm{s}^{-1}\right)\end{array}$ & $\begin{array}{l}\text { 饱和光强 } \\
I_{\mathrm{sat}} \\
\left(\mu \mathrm{mol} \cdot \mathrm{m}^{-2} \cdot \mathrm{s}^{-1}\right)\end{array}$ & $\begin{array}{l}\text { 光补偿点 } \\
I_{\mathrm{c}} \\
\left(\mu \mathrm{mol} \cdot \mathrm{m}^{-2} \cdot \mathrm{s}^{-1}\right)\end{array}$ & $\begin{array}{l}\text { 暗呼吸速率 } \\
R_{\mathrm{d}} \\
\left(\mu \mathrm{mol} \cdot \mathrm{m}^{-2} \cdot \mathrm{s}^{-1}\right)\end{array}$ & $\begin{array}{l}\text { 决定系数 } \\
\text { Determina- } \\
\text { tion coeffi- } \\
\text { cient }\end{array}$ \\
\hline & $\begin{array}{l}\text { 上部叶 } \\
\text { Upper leaf }\end{array}$ & $\begin{array}{l}\text { 直角双曲线 } \\
\text { Rectangurar hyperbolic }\end{array}$ & $0.083^{\mathrm{a}}$ & $55.89^{\text {Aa }}$ & $997^{\mathrm{Aa}}$ & $48.35^{\mathrm{Aa}}$ & $3.70^{\mathrm{Aa}}$ & 0.998 \\
\hline & & $\begin{array}{l}\text { 非直角双曲线 } \\
\text { Non-rectangular hyperbolic }\end{array}$ & $0.064^{\mathrm{b}}$ & $48.70^{\mathrm{Ab}}$ & $846^{\mathrm{Ab}}$ & $49.33^{\mathrm{Aa}}$ & $3.06^{\mathrm{Ab}}$ & 0.998 \\
\hline & & $\begin{array}{l}\text { 叶子飘模型 } \\
\text { Ye Zi-Piao model }\end{array}$ & $0.068^{\mathrm{c}}$ & $37.12^{\mathrm{Bc}}$ & $2235^{\mathrm{Bc}}$ & $48.71^{\mathrm{Aa}}$ & $3.15^{\mathrm{Ab}}$ & 0.999 \\
\hline & & $\begin{array}{l}\text { 测量值 } \\
\text { Measured data }\end{array}$ & - & $36.78^{\mathrm{B}}$ & $2000^{\mathrm{B}}$ & $48.87^{\mathrm{A}}$ & $3.36^{\mathrm{B}}$ & - \\
\hline & & $\begin{array}{l}\text { 直角双曲线 } \\
\text { Rectangular hyperbolic }\end{array}$ & $0.079^{\mathrm{a}}$ & $57.44^{\mathrm{Aa}}$ & $1071^{\mathrm{Aa}}$ & $50.01^{\mathrm{Aa}}$ & $3.65^{\mathrm{Aa}}$ & 0.997 \\
\hline \multirow{12}{*}{$\begin{array}{l}\text { 大喇叭口期 } \\
\text { Bell stage }\end{array}$} & 穗位叶 & 非直角双曲线 & $0.057^{\mathrm{b}}$ & $47.35^{\mathrm{Ab}}$ & $880^{\mathrm{Ab}}$ & $50.75^{\mathrm{Aa}}$ & $2.83^{\mathrm{Ab}}$ & 0.998 \\
\hline & $\begin{array}{l}\text { Lear at } \\
\text { ear of } \\
\text { grain }\end{array}$ & $\begin{array}{l}\text { Non-rectangular hyperbolic } \\
\text { 叶子飘模型 } \\
\text { Ye Zi-Piao model }\end{array}$ & $0.063^{\mathrm{c}}$ & $37.10^{\mathrm{BC}}$ & $2061^{\mathrm{Ac}}$ & $50.23^{\mathrm{Aa}}$ & $3.01^{\mathrm{Ac}}$ & 0.999 \\
\hline & & $\begin{array}{l}\text { 测量值 } \\
\text { Measured data }\end{array}$ & - & $36.99^{\mathrm{B}}$ & $2000^{\mathrm{B}}$ & $49.14^{\mathrm{A}}$ & $3.25^{\mathrm{B}}$ & - \\
\hline & & $\begin{array}{l}\text { 直角双曲线 } \\
\text { Rectangular hyperbolic }\end{array}$ & $0.090^{\mathrm{a}}$ & $33.93^{\mathrm{Aa}}$ & $729^{\mathrm{Aa}}$ & $41.46^{\mathrm{Aa}}$ & $3.27^{\text {Aa }}$ & 0.996 \\
\hline & $\begin{array}{l}\text { 下部叶 } \\
\text { Lower leaf }\end{array}$ & $\begin{array}{l}\text { 非直角双曲线 } \\
\text { Non-rectangular hyperbolic }\end{array}$ & $0.059^{\mathrm{b}}$ & $29.56^{\mathrm{Ab}}$ & $628^{\mathrm{Ab}}$ & $44.14^{\mathrm{Aa}}$ & $2.50^{\mathrm{Ba}}$ & 0.999 \\
\hline & & $\begin{array}{l}\text { 叶子飘模型 } \\
\text { Ye Zi-Piao model }\end{array}$ & $0.070^{\mathrm{c}}$ & $24.18^{\mathrm{Bc}}$ & $1789^{\mathrm{Bc}}$ & $42.98^{\mathrm{Aa}}$ & $2.76^{\mathrm{Bb}}$ & 0.999 \\
\hline & & $\begin{array}{l}\text { 测量值 } \\
\text { Measured data }\end{array}$ & - & $24.20^{\mathrm{B}}$ & $1800^{\mathrm{B}}$ & $43.40^{\mathrm{A}}$ & $2.63^{\mathrm{B}}$ & - \\
\hline & & $\begin{array}{l}\text { 直角双曲线 } \\
\text { Rectangular hyperbolic }\end{array}$ & $0.102^{\mathrm{a}}$ & $25.30^{\mathrm{Aa}}$ & $540^{\mathrm{Aa}}$ & $31.31^{\mathrm{Aa}}$ & $2.71^{\mathrm{Aa}}$ & 0.997 \\
\hline & 上部叶 & $\begin{array}{l}\text { 非直角双曲线 } \\
\text { Non-rectangular hyperbolic }\end{array}$ & $0.076^{\mathrm{b}}$ & $23.90^{\mathrm{Bb}}$ & $499^{\mathrm{Aa}}$ & $32.29^{\mathrm{Aa}}$ & $2.29^{\mathrm{Bb}}$ & 0.999 \\
\hline & Upper leaf & $\begin{array}{l}\text { 叶子飘模型 } \\
\text { Ye Zi-Piao model }\end{array}$ & $0.089^{c}$ & $22.71^{\mathrm{Bb}}$ & $1973^{\mathrm{Bb}}$ & $31.63^{\mathrm{Aa}}$ & $2.45^{\mathrm{Ba}}$ & 0.998 \\
\hline & & $\begin{array}{l}\text { 测量值 } \\
\text { Measured data }\end{array}$ & - & $19.70^{\mathrm{B}}$ & $1800^{\mathrm{B}}$ & $32.29^{\mathrm{Aa}}$ & $2.24^{\mathrm{B}}$ & - \\
\hline & & $\begin{array}{l}\text { 直角双曲线 } \\
\text { Rectangular hyperbolic }\end{array}$ & $0.076^{\mathrm{a}}$ & $24.94^{\mathrm{Aa}}$ & $645^{\mathrm{Aa}}$ & $32.09^{\mathrm{Aa}}$ & $2.16^{\mathrm{Aa}}$ & 0.996 \\
\hline \multirow{7}{*}{$\begin{array}{l}\text { 乳熟期 } \\
\text { Milk stage }\end{array}$} & 穗位叶 & 非直角双曲线 & $0.050^{\mathrm{b}}$ & $20.02^{\mathrm{Ab}}$ & $561^{\mathrm{Ab}}$ & $32.88^{\mathrm{Aa}}$ & $1.59^{\mathrm{Bb}}$ & 0.998 \\
\hline & $\begin{array}{l}\text { ear of } \\
\text { grain }\end{array}$ & $\begin{array}{l}\text { 叶子飘模型 } \\
\text { Ye Zi-Piao model }\end{array}$ & $0.062^{c}$ & $18.55^{\mathrm{Bc}}$ & $1819^{\mathrm{Bc}}$ & $32.36^{\mathrm{Aa}}$ & $1.83^{\mathrm{Bb}}$ & 0.999 \\
\hline & & $\begin{array}{l}\text { 测量值 } \\
\text { Measured data }\end{array}$ & - & $18.66^{\mathrm{B}}$ & $1800^{\mathrm{B}}$ & $32.32^{\mathrm{A}}$ & $1.74^{\mathrm{B}}$ & - \\
\hline & & $\begin{array}{l}\text { 直角双曲线 } \\
\text { Rectangular hyperbolic }\end{array}$ & $0.073^{\mathrm{a}}$ & $21.88^{\mathrm{Aa}}$ & $595^{\mathrm{Aa}}$ & $30.41^{\mathrm{Aa}}$ & $1.92^{\mathrm{Aa}}$ & 0.996 \\
\hline & $\begin{array}{l}\text { 下部叶 } \\
\text { Lower leaf }\end{array}$ & $\begin{array}{l}\text { 非直角双曲线 } \\
\text { Non-rectangular hyperbolic }\end{array}$ & $0.049^{\mathrm{b}}$ & $19.67^{\mathrm{Ab}}$ & $528^{\mathrm{Ab}}$ & $30.81^{\mathrm{Aa}}$ & $1.45^{\mathrm{Bb}}$ & 0.998 \\
\hline & & $\begin{array}{l}\text { 叶子飘模型 } \\
\text { Ye Zi-Piao model }\end{array}$ & $0.059^{c}$ & $16.45^{\mathrm{Bc}}$ & $1777^{\mathrm{Bc}}$ & $30.40^{\mathrm{Aa}}$ & $1.61^{\mathrm{Bb}}$ & 0.999 \\
\hline & & $\begin{array}{l}\text { 测量值 } \\
\text { Measured data }\end{array}$ & - & $16.44^{\mathrm{B}}$ & $1800^{\mathrm{B}}$ & $31.43^{\mathrm{A}}$ & $1.50^{\mathrm{B}}$ & - \\
\hline
\end{tabular}

根据最小显著差异法, 不同小写字母表示3种模型的拟合值在 0.05 显著水平下差异显著, 不同大写字母表示各模型拟合值与实测值在0.05显著水平下差 异显著。

Different lowercase letters indicate significant difference at 0.05 level among three models; different uppercase letters indicate significant difference at 0.05 level between fitted value and measured data according to least significant difference. $\alpha$, initial quantum efficiency; the rest of notes see Table 1 .

型的拟合值均远大于实测值, 但与实测值的偏离度 是不一样的，直角双曲线模型在大喇叭口期上部
叶、穗位叶、下部叶较实测值的偏离度依次为 $52 \%$ 、 $55 \% 、 26 \%$ ，大喇叭口期依次为40\%、34\%、33\%; 非 

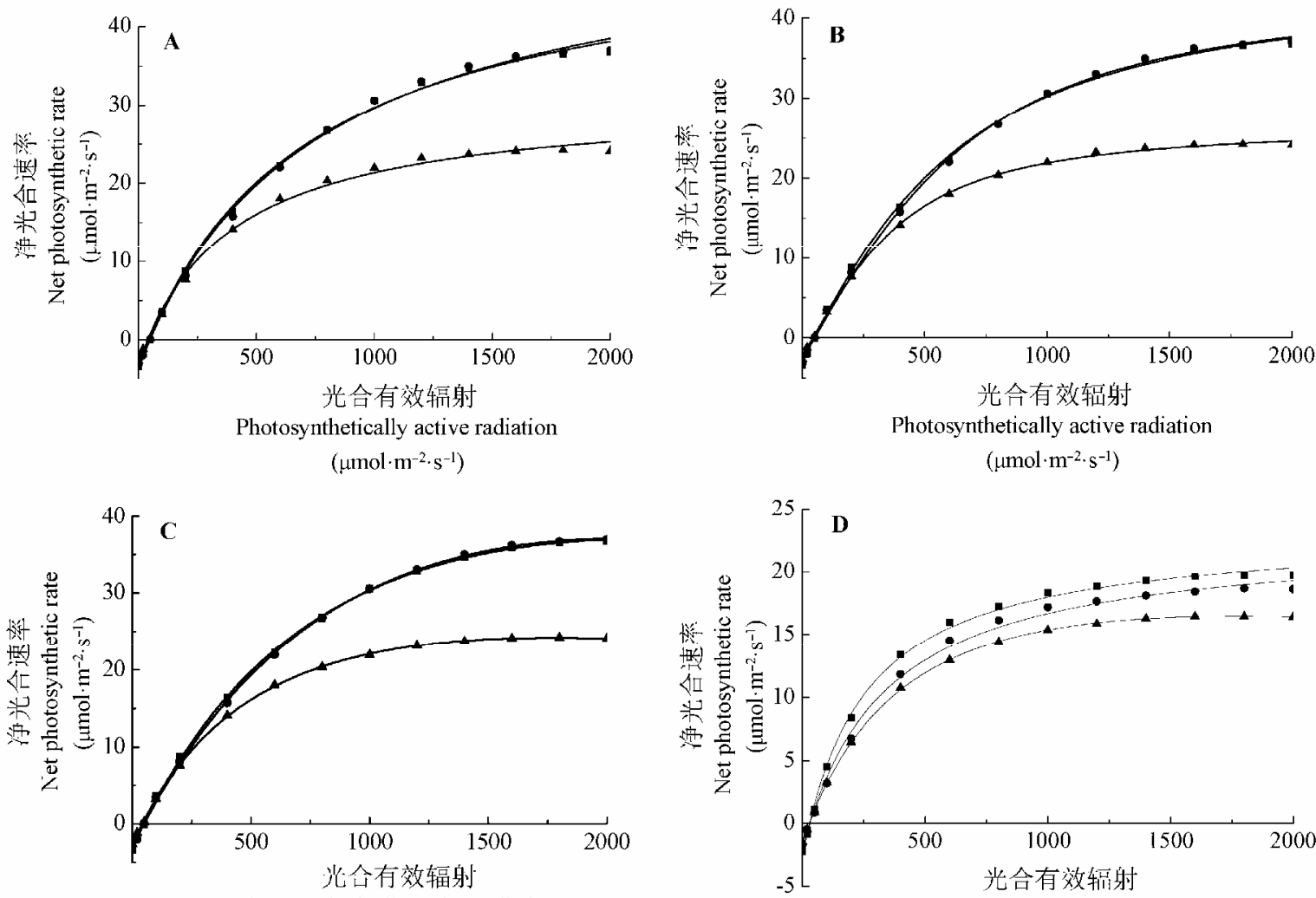

Photosynthetically active radiation

$\left(\mu \mathrm{mol} \cdot \mathrm{m}^{-2} \cdot \mathrm{s}^{-1}\right)$

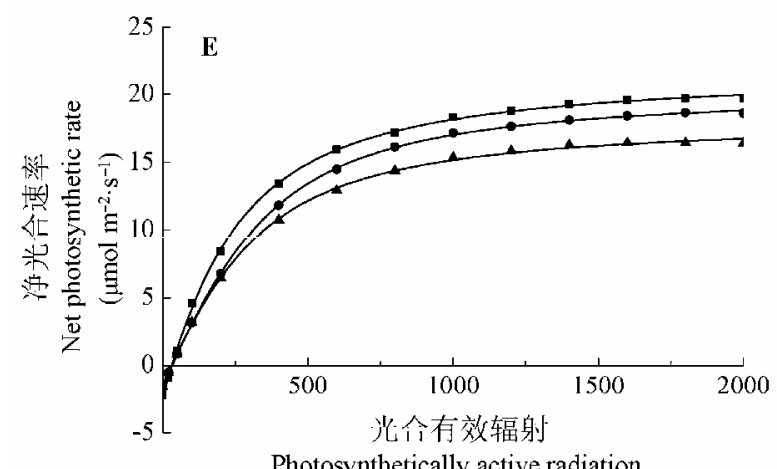

Photosynthetically active radiation $\left(\mu \mathrm{mol} \cdot \mathrm{m}^{-2} \cdot \mathrm{s}^{-1}\right)$

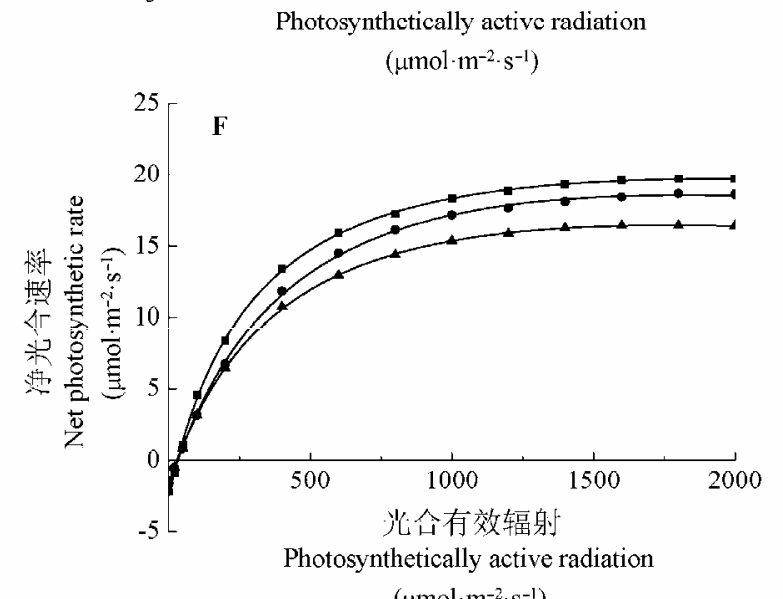

- 上部叶 Upper leaf - 穗位叶 Leaf at ear of grain $\boldsymbol{\Delta}$ 下部叶 Lower leaf - 3 种模型的拟合曲线 Light response curves fitted by three models

图1 夏玉米大喇叭口期与乳熟期 3 种模型的光合-光响应曲线拟合图。 $\mathbf{A}$, 直角双曲线模型对大喇叭口期叶片的光合-光响应曲 线。B, 非直角双曲线模型对大喇叭口期叶片的光合-光响应曲线。C, 叶子飘模型对大喇叭口期叶片的光合-光响应曲线。D, 直 角双曲线模型对乳熟期叶片的光合-光响应曲线。 $\mathbf{E}$, 非直角双曲线模型对乳熟期叶片的光合-光响应曲线。 $\mathbf{F}$, 叶子飘模型对 乳熟期叶片的光合-光响应曲线。

Fig. 1 Light response curves fitted by three models at bell stage and milk stage for summer maize. A, Light response curves fitted by rectangular hyperbolic model at bell stage. B, Light response curves fitted by non-rectangular hyperbolic model at bell stage. C, Light response curves fitted by Ye Zi-Piao model at bell stage. D, Light response curves fitted by rectangular hyperbolic model at milk stage. E, Light response curves fitted by non-rectangular hyperbolic model at milk stage. F, Light response curves fitted by Ye Zi-Piao model at milk stage.

直角双曲线模型在大喇叭口期的偏离度依次为 $32 \% 、 28 \% 、 22 \%$, 乳熟期依次为 $21 \% 、 7 \% 、 20 \%$, 可 以看出乳熟期的偏离度整体是小于大喇叭口期的,
叶片由上而下偏离度也是降低的。对 $I_{\mathrm{sat}}$ 的拟合值叶 子飘模型与实测值最接近, 直角双曲线模型与非直 角双曲线模型远小于实测值, 直角双曲线模型在大 
喇叭口期上部叶、穗位叶、下部叶的偏离度依次为 $50 \% 、 46 \% 、 60 \%$, 在乳熟期的偏离度依次为 $58 \%$ 、 $56 \% 、 65 \%$; 非直角双曲线模型在大喇叭口期的偏离 度依次为70\%、64\%、67\%, 乳熟期的偏离度依次为 $72 \% 、 69 \% 、 70 \%$, 乳熟期的偏差整体大于大喇叭口 期, 不同部位叶片之间无明显差异。3种模型对光补 偿点的拟合结果均较好。直角双曲线模型对 $R_{\mathrm{d}}$ 的拟 合值均大于实测值, 在大喇叭口期上部叶、穗位叶、 下部叶较实测值的偏离度依次为 $10 \% 、 12 \% 、 24 \%$, 乳熟期依次为 $21 \% 、 24 \% 、 28 \%$, 乳熟期的偏离度整 体上大于大喇叭口期的, 叶片由上而下偏离度也是 增加的; 非直角双曲线模型和叶子飘模型对乳熟期 $R_{\mathrm{d}}$ 的拟合整体较好, 而在大喇叭口期只对呼吸作用 相对较弱的下部叶可以较好地拟合。从各拟合参数 来看, 对于光补偿点, 3 种模型均可以较好地反映实 测值; 对于 $R_{\mathrm{d}}$, 非直角双曲线模型和叶子飘模型优 于直角双曲线模型; 对于 $P_{\mathrm{nmax}}$ 和 $I_{\mathrm{sat}}$, 叶子飘模型优 于其余两种模型, 这也反映了决定系数高只能说明 曲线拟合程度好, 并不能保证拟合结果一定与实测 值相符。

从拟合图(图1)可以看出: 各模型对两生育期三 部位的叶片均是可以拟合的, 拟合图更加直观地表 现出两时期各部位叶片的光合情况: 大喇叭口期上 部叶与穗位叶的拟合曲线基本重合, 上部叶净光合 速率大于下部叶, 乳熟期净光合速率上部叶 $>$ 穗位 叶>下部叶, 大喇叭口期曲线变平缓的光强点相对 于乳熟期明显靠后, 表明大喇叭口期的 $I_{\mathrm{sat}}$ 大于乳熟 期, 曲线所表现出的规律与上文对实测数据的分析 结果是一致的。这是由于叶龄的原因, 在大喇叭口 期, 玉米上部叶和穗位叶均较新, 光合作用强烈, 拟合图中便出现上部叶与穗位叶的拟合曲线重合且 整体位于下部叶拟合曲线的上方, 而到了乳熟期, 三处叶片均开始衰老, 但上部叶较穗位叶叶龄小, 穗位叶较下部叶叶龄小, 图中便出现由上而下光合 递减的阶梯状分布, 大喇叭口期的叶片比乳熟期要 新, 其净光合速率整体便大于乳熟期。

图1中各拟合曲线的坡度均由陡变缓, 依据光 强分为坡度较大阶段 $\left(0-200 \mu \mathrm{mol} \cdot \mathrm{m}^{-2} \cdot \mathrm{s}^{-1}\right)$ 、过渡阶 段(400-1 $\left.000 \mu \mathrm{mol} \cdot \mathrm{m}^{-2} \cdot \mathrm{s}^{-1}\right)$ 和平缓阶段(1 200-2 000 $\left.\mu \mathrm{mol} \cdot \mathrm{m}^{-2} \cdot \mathrm{s}^{-1}\right)$ 。在坡度较大阶段, 净光合速率快速增 高, 3 种模型的拟合曲线均可以较好地穿过实测点, 过渡阶段曲线处于一个由陡变缓的过程, 说明净光
合速率仍在增加, 但增速放缓, 图1中直角双曲线模 型有部分实测点没有落在拟合曲线上, 平缓阶段曲 线坡度变平缓, 净光合速率基本不变, 直角双曲线 模型与非直角双曲线模型均有部分点没有落在拟合 曲线上, 非直角双曲线模型优于直角双曲线模型。 对此, 分别求取所有拟合曲线在坡度较大阶段、过 渡阶段和平缓阶段的残差平方和, 结果见表3。在坡 度较大阶段, 3 种模型的残差平方和均较小, 此阶段 对光合特征参数(光补偿点、暗呼吸速率)的拟合(表 2)也均较好; 过渡阶段的残差平方和只有直角双曲 线模型大于 1 , 在图1中表现出部分实测数据没有落 在拟合曲线上; 平缓阶段高光部分叶子飘模型的残 差最小, 对应的实测值对拟合曲线的符合度(图1)和 对光合特征参数 $\left(P_{\mathrm{nmax}} 、 I_{\mathrm{sat}}\right)$ 的拟合 $($ 表 2$)$ 均为最优。

表3 3种模型拟合曲线的残差平方和

Table 3 Residual sum of squares of light-response curves fitted by three models

\begin{tabular}{llll}
\hline $\begin{array}{l}\text { 光合有效辐射 } P A R \\
\left(\mu \mathrm{mol} \cdot \mathrm{m}^{-2} \cdot \mathrm{s}^{-1}\right)\end{array}$ & $0-200$ & $400-1000$ & $1200-2000$ \\
\hline $\begin{array}{l}\text { 直角双曲线 } \\
\begin{array}{l}\text { Rectangular hyperbolic } \\
\text { 非直角双曲线 }\end{array}\end{array}$ & 0.75 & 1.15 & 1.92 \\
$\begin{array}{l}\text { Non-rectangular hyperbolic } \\
\text { 叶子飘模型 }\end{array}$ & 0.40 & 0.31 & 0.50 \\
\begin{tabular}{l} 
Ye Zi-Piao model \\
\hline
\end{tabular} & 0.28 & 0.14 & 0.05 \\
\hline
\end{tabular}

PAR, photosynthetically active radiation.

\section{3 讨论}

随着叶龄增加, 叶片逐渐衰老, 气孔导度下降, Rubisco 含量和活性开始下降, 光合电子传递能力 降低, 因而光合能力逐渐减弱(Dai et al., 2004)。无 论哪种模型拟合均可以反映出夏玉米在叶龄的影响 下随着叶位靠下与生育期的靠后, 净光合速率会下 降并随 $P A R$ 变化的拐点会靠前。在 $P A R$ 为 $0-2000$ $\mu \mathrm{mol} \cdot \mathrm{m}^{-2} \cdot \mathrm{s}^{-1}$ 时, 光合特性的变化是有一定规律可 循的, 在大喇叭口期 $P A R$ 为 $0-200 \mu \mathrm{mol} \cdot \mathrm{m}^{-2} \cdot \mathrm{s}^{-1}$ 时, 3 个部位叶片的净光合速率基本一致, 而乳熟期 $P A R$ 为 $0-100 \mu \mathrm{mol} \cdot \mathrm{m}^{-2} \cdot \mathrm{s}^{-1}$ 时, 3 个部位叶片的净光合速率 基本一致。以此为分界点, 随着 $P A R$ 的增大, 大喇叭 口期上部叶与穗位叶净光合速率的增速明显大于下 部叶, 乳熟期净光合速率的增速为上部叶 $>$ 穗位叶 $>$ 下部叶。大喇叭口期上部叶与穗位叶在 $P A R$ 为 1400 $\mu \mathrm{mol} \cdot \mathrm{m}^{-2} \cdot \mathrm{s}^{-1}$ 时, 净光合速率的增速放缓, 下部叶在 $P A R$ 为 $1000 \mu \mathrm{mol} \cdot \mathrm{m}^{-2} \cdot \mathrm{s}^{-1}$ 时增速放缓; 乳熟期净光 
合速率均在 $P A R$ 为 $1000 \mu \mathrm{mol} \cdot \mathrm{m}^{-2} \cdot \mathrm{s}^{-1}$ 时增速放缓。任 丽丽和高辉远(2008)对酸模(Rumex acetosa)、师生波 等(2011)对不同叶龄拟南芥(Arabidopsis thaliana)的 研究均表明叶片充分生长后随着叶龄的增长植株光 合能力下降, 本研究中玉米乳熟期光合能力小于大 喇叭口期, 叶位由上而下光合能力逐步降低, 与他 们的研究结论一致。叶龄是影响植株暗呼吸的一个 主要因素, 叶片的 $R_{\mathrm{d}}$ 均随着叶龄的增加而降低 (Villar et al., 1995; 孙金伟等, 2013), 这是因为快速 生长期的植株需要更多的物质和能量, 以满足生长 需求, 大喇叭口期叶片比乳熟期叶片新, 越靠上部 叶位的叶龄越小, 随着生育期的靠后以及叶位由上 而下, $R_{\mathrm{d}}$ 逐步降低。AQE与 $R_{\mathrm{d}}$ 呈现相同的变化规律。 我们综合各光合特征参数的变化规律可以发现, 其 与拟合曲线在坡度较大阶段是吻合的, 因为新叶片 的 $A Q E$ 高, 其在低光强阶段的光合作用强, 但其暗 呼吸作用也强, 较老的叶片 $A Q E$ 低暗呼吸也弱, 在 两者的共同作用下拟合曲线在低光阶段便出现重合 的现象; 随着光强的升高, 光合作用速率增加, 暗 呼吸的影响相对减弱, 不同叶龄的拟合曲线在坡度 较大阶段后便呈现不同变化趋势。

对于植物光合-光响应曲线的拟合, 常见的模 型有直角双曲线模型、非直角双曲线模型、指数函 数模型和叶子飘模型, 指数函数模型对于玉米等 $\mathrm{C}_{4}$ 植物应用较少, 本文中并没有采用。Yu等(2004)关 于冬小麦(Triticum aestivum)气孔导度与叶片光合、 蒸腾等关系的研究表明直角双曲线模型极大地估算 了叶片的 $P_{\text {nmax }}$; Junior等(2013)对亚马孙河10种树木 进行了大量的光响应曲线拟合, 表明直角双曲线与 非直角双曲线模型会过大估算 $P_{\text {nmax }}$; Lobo等(2013) 用Microsoft Excel拟合光合-光响应曲线时对比分析 了各拟合模型, 肯定了叶子飘模型在 $P_{\mathrm{nmax}}$ 和 $I_{\mathrm{sat}}$ 方面 拟合的优势。本文的结论与上述研究成果是一致的, 但Lobo等(2013)的研究结果同时也表明该模型对模 型各参数的生理学意义没有作深远的阐述。这一问 题现在已解决, $\beta$ 为光抑制项, $\gamma$ 为光饱和项, 当 $\gamma$ 一 定时, $\beta$ 值越大, 光响应曲线弯曲程度就越大, 植物 受光抑制就越强。当 $\beta$ 一定时, $\gamma$ 值越小, 表示光响应 曲线弯曲程度就越小, 植物就越不容易发生光饱和 现象(叶子飘和康华靖, 2012)。然而, 各模型的差异 在不同生育期不同部位叶片之间是否有所变化, 前 人并没有给出结果, 通过对拟合参数与实测数据的
对比, 发现对于 $P_{n \max }$, 直角双曲线模型与非直角双 曲线模型与实测数据的偏离度在大喇叭口期上部叶 与穗位叶一致, 而到下部叶是降低的, 乳熟期的偏 离度上部叶>穗位叶>下部叶, 大喇叭口期的偏差整 体大于乳熟期, 与净光合速率的变化是一致的, 光 合作用越强, 偏离度越大; 对 $I_{\mathrm{sat}}$ 拟合的偏离情况不 同部位叶片之间未发现统一规律, 乳熟期整体大于 大喇叭口期, 叶子飘模型在不同生育期不同叶位拟 合中并无这种偏差的情况, 有很好的稳定性, 与实 测值也最为接近。从拟合图形与残差分析可以看出, 3 种模型对于 $0-200 \mu \mathrm{mol} \cdot \mathrm{m}^{-2} \cdot \mathrm{s}^{-1}$ 低光强阶段的拟合 均较好, 与表 2 中 3 种模型对低光阶段的暗呼吸速率 与光补偿点较好的拟合是相对应的; $P A R$ 为 400-1 $000 \mu \mathrm{mol} \cdot \mathrm{m}^{-2} \cdot \mathrm{s}^{-1}$ 时, 直角双曲线模型实测点 与拟合曲线偏离较大; 对 $1200-2000 \mu \mathrm{mol} \cdot \mathrm{m}^{-2} \cdot \mathrm{s}^{-1}$ 高光强部分, 直角双曲线模型和非直角双曲线模型 的拟合曲线与光合特征参数 $\left(I_{\mathrm{sat}} 、 P_{\mathrm{nmax}}\right)$ 均与实测值 有所差异, 拟合残差大于叶子飘模型, 这是由模型 的机制所决定的, 由各模型的公式可以知道, 直角 双曲线模型与非直角双曲线模型均是没有极值的方 程, 所以只能拟合植物不存在光抑制时的光响应曲 线; 叶子飘模型便体现出它独有的优越性, 它存在 极值, 可以在不做任何假设的情况下由方程本身直 接求出 $P_{\mathrm{nmax}}$ 与 $I_{\mathrm{sat}}$, 文中在此基础上, 用该模型对夏 玉米叶片做光合-光响应曲线拟合, 结合残差分析, 表明该模型对夏玉米叶片0-2 $000 \mu \mathrm{mol} \cdot \mathrm{m}^{-2} \cdot \mathrm{s}^{-1}$ 各阶 段光强均有较好的适应性。

\section{基金项目 国家自然科学基金(51409006)。}

\section{参考文献}

Baly EC (1935). The kinetics of photosynthesis. Proceedings of the Royal Society of London Series B, Biological Sciences, 117, 218-239.

Bassman JH, Zwier JC (1991). Gas exchange characteristics of Populus trichocarpa, Populus deltoides and Populus trichocarpa $\times$ P. deltoides. Tree Physiology, 8, 145-159.

Chen HH, Jiang BL, Liu C, Chen WQ, Lu Y, Zhang S (2013). Image super-resolution reconstruction based on residual error. Journal of Image and Graphics, (1), 42-48. (in Chinese with English abstract) [陈华华, 姜宝林, 刘超, 陈伟 强, 陆宇, 张嵩 (2013). 基于残差的图像超分辨率重建. 中国图象图形学报, (1), 42-48.]

Chen KL, Li JM, He HQ, H XH, Yao YZ, Sun SJ (2013). Effects of water on photosynthesis in different age of tomato 
leaves. Acta Ecologica Sinica, 33, 4919-4929. (in Chinese with English abstract) [陈凯利, 李建明, 贺会强, 胡晓 辉, 姚勇哲, 孙三杰 (2013). 水分对番茄不同叶龄叶片 光合作用的影响. 生态学报, 33, 4919-4929.]

Dai J, Gao H, Dai Y, Zou Q (2004). Changes in activity of energy dissipating mechanisms in wheat flag leaves during senescence. Plant Biology, 6, 171-177.

Jiang GM (2004). Plant Ecophysiology. Higher Education Press, Beijing. (in Chinese) [蒋高明 (2004). 植物生理生 态学. 高等教育出版社, 北京.]

Junior UMDS, Gonçalves JFDC, Fearnside PM (2013). Measuring the impact of flooding on Amazonian trees: Photosynthetic response models for ten species flooded by hydroelectric dams. Trees, 27, 193-210.

Lang Y, Zhang GC, Zhang ZK, Liu SS, Liu DH, Hu XL (2011). Light response of photosynthesis and its simulation in leaves of Prunus sibirica L. under different soil water conditions. Acta Ecologica Sinica, 31, 4499-4508. (in Chinese with English abstract) [郎荣, 张光灿, 张征坤, 刘顺生, 刘德虎, 胡小兰 (2011). 不同土壤水分下山杏光合作用 光响应过程及其模拟. 生态学报, 31, 4499-4508.]

Liu YF, Xiao LT, Tong JH, Li XB (2005). Primary application on the non-rectangular hyperbola model for photosynthetic light-response curve. Chinese Agricultural Science Bulletin, 21(8), 76-79. (in Chinese with English abstract) [刘宇 锋, 萧浪涛, 童建华, 李晓波 (2005). 非直线双曲线模 型在光合光响应曲线数据分析中的应用. 中国农学通 报, 21(8), 76-79.]

Lobo FDA, Barros MPD, Dalmagro HJ, Dalmolin ÂC (2013). Fitting net photosynthetic light-response curves with Microsoft Excel - A critical look at the models. Photosynthetica, 51, 445-456.

Lu PL, Luo Y, Liu JD, Yu Q (2000). Characteristic parameters of light response curves of photosynthesis of winter wheat in north China. Quarterly Journal of Applied Meteorology, 11, 236-241. (in Chinese with English abstract) [陆佩玲, 罗毅, 刘建栋, 于强 (2000). 华北地区冬小麦光合作用 的光响应曲线的特征参数. 应用气象学报, 11 , 236-241.]

Ren LL, Gao HY (2008). Effects of $\mathrm{NaCl}$ stress on induction of photosynthesis and PSII photochemistry efficiency of $R u$ mex K-1 leaves with different age. Acta Botanica Boreali-Occidentalia Sinica, 28, 1014-1019. (in Chinese with English abstract) [任丽丽, 高辉远 (2008). 不同叶龄杂 交酸模叶片光合速率及PSII光化学效率诱导对 $\mathrm{NaCl}$ 胁 迫的响应. 西北植物学报, 28, 1014-1019.]

Shi SB, Neil B, Tracy L, Zhang DG (2011). Analysis of chlorophyll fluorescence parameters in Arabidopsis thaliana leaves of different ages and its response to strong light. Grass and Turf, 31(4), 51-55. (in Chinese with English abstract) [师生波, Neil B, Tracy L, 张德罡 (2011). 不同
叶龄拟南芥苂光参数分析及对强光的响应. 草原与草 坪, 31(4), 51-55.]

Sun JW, Yuan FH, Guan DX, Wu JB (2013). Dark respiration of terrestrial vegetations: A review. Chinese Journal of Applied Ecology, 24, 1739-1746. (in Chinese with English abstract) [孙金伟, 袁凤辉, 关德新, 吴家兵 (2013). 陆 地植被暗呼吸的研究进展。应用生态学报, 24, 1739-1746.]

Sun LQ, Hao ZC, Wang JH, Ioan N, Ousmone S (2014). Assessment and correction of TMPA products 3B43RT and 3B42V6. Journal of Hydraulic Engineering, 45, 11351146. (in Chinese with English abstract) [孙乐强, 郝振纯, 王加虎, Ioan N, Ousmone S (2014). TMPA卫星降水数据 的评估与校正. 水利学报, 45, 1135-1146.]

Thornley JHM (1976). Mathematical Models in Plant Physiology. Academic Press, London.

Villar R, Held AA, Merino J (1995). Dark leaf respiration in light and darkness of an evergreen and a deciduous plant species. Plant Physiology, 107, 421-427.

Wang ZL, Yang C, Du JC, Hu HF, Zhao LL, Mao XT (2009). Photosynthetic characteristics and photo-adaptability of four Melilotodes ruthenica ecotypes. Chinese Journal of Ecology, 28, 1035-1040. (in Chinese with English abstract) [王照兰, 杨持, 杜建材, 胡卉芳, 赵丽丽, 毛小 涛 (2009). 不同生态型扁宿豆光合特性和光适应能力. 生态学杂志, 28, 1035-1040.]

Wei YF, Fang J, Zhao XY, Li SG (2011). Eco-physiological traits of different-age needles of Pinus sylvestris var. mongolica plantation in Horqin sandy land of China. Chinese Journal of Plant Ecology, 35, 1271-1280. (in Chinese with English abstract) [魏雅芬, 方杰, 赵学勇, 李胜功 (2011). 科尔沁沙地樟子松人工林不同年龄针 叶生理生态性状. 植物生态学报, 35, 1271-1280.]

Ye ZP (2007). A new model for relationship between irradiance and the rate of photosynthesis in Oryza sativa. Photosynthetica, 45, 637-640.

Ye ZP (2010). A review on modeling of responses of photosynthesis to light and $\mathrm{CO}_{2}$. Chinese Journal of Plant Ecology, 34, 727-740. (in Chinese with English abstract) [叶子 飘 (2010). 光合作用对光和 $\mathrm{CO}_{2}$ 响应模型的研究进展. 植物生态学报, 34, 727-740.]

Ye ZP, Kang HJ (2012). Study on biological significance of coefficient in modified model of photosynthesisirradiance. Journal of Yangzhou University (Agricultural and Life Science Edition), 33(2), 51-57. (in Chinese with English abstract) [叶子飘, 康华靖 (2012). 植物光响应 修正模型中系数的生物学意义研究. 扬州大学学报(农 业与生命科学版), 33 (2), 51-57.]

Yu Q, Zhang Y, Liu Y, Shi P (2004). Simulation of the stomatal conductance of winter wheat in response to light, temperature and $\mathrm{CO}_{2}$ changes. Annals of Botany, 93, 
435-441.

Zhang QD, Jiang GM, Zhu XG, Wang Q, Lu CM, Bai KZ, Kuang TY (2001). Photosynthetic capability of 12 genotypes of Triticum aestivum. Acta Phytoecologica Sinica, 25, 532-536. (in Chinese with English abstract) [张其德, 蒋高明, 朱新广, 王强, 卢从明, 白克智, 匡廷云 (2001). 12个不同基因型冬小麦的光合能力. 植物生态 学报, 25, 532-536.]

Zhong C, Zhang MD, Hu XQ, Zhu Y (2012). Effects of temperature variation on the light-response characteristics of tobacco leaf photosynthesis. Chinese Journal of Ecology, 31, 337-341. (in Chinese with English abstract) [钟楚, 张
明达, 胡雪琼, 朱勇 (2012). 温度变化对烟草光合作用 光响应特征的影响. 生态学杂志, 31, 337-341.]

Zhou P, Wang XL, Ma YX, Ma HT, Xu XJ, Liu ZJ (2010). Analysis on residual error for adaptive optical system based on stochastic parallel gradient descent control algorithm. Acta Optica Sinica, 30, 613-617. (in Chinese with English abstract) [周朴, 王小林, 马阎星, 马浩统, 许晓 军, 刘泽金 (2010). 基于随机并行梯度下降算法自适应 光学系统的校正残差分析. 光学学报, 30, 613-617.]

特邀编委: 叶子飘 责任编辑: 李 敏

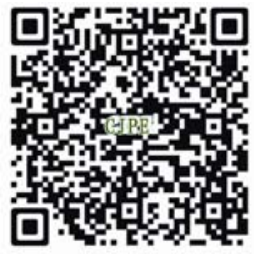

植物生态学报官网

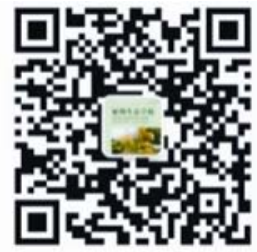

微信订阅号

期刊及学科

相关信息发布

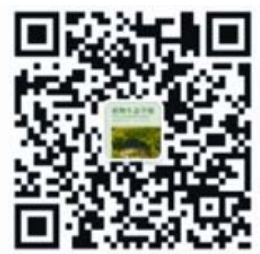

微信服务号

稿件状态查询

全文检索汶览 